\title{
Posterior wall acetabular osteotomy for removal of a juxta-articular bullet
}

\begin{abstract}
Complications following retained intra and juxta-articular bullets and bullet fragments include mechanical blockade to movement, direct cartilage destruction, joint sepsis, lead arthropathy and even systemic lead poisoning (plumbism). It is therefore recommended that all intra and juxta-articular bullets or bullet fragments should be removed. Removal can be performed via open arthrotomy or arthroscopically. Occasionally the location requires the use of ingenious techniques.

The authors present a case of a patient who presented with a single gunshot wound with an entry in the right suprapubic area, with no exit wound. Radiographs and a CT scan revealed a bullet tract that traversed the quadrilateral surface of the acetabulum with the bullet lodged juxta-articularly in the posterior acetabular wall of the left hip.

For bullet removal the authors did not consider approaches through the joint, arthroscopic or open, as the bullet was buried sub-chondrally. It was decided to perform an osteotomy of the posterior wall as this would minimize damage to the acetabular articular cartilage and the capsule would be left undisturbed. This was achieved via an osteotomy through a Kocher-Langenbeck approach to the acetabulum. The bullet was removed and the osteotomy reduced and fixed with two interfragmentary screws and a buttress plate. A standard post-operative regime for posterior wall fixation was followed.

At 2 years follow up the osteotomy site had united with no complications and the patient had regained a pain-free full range of motion, was able to walk unaided and had no limp. The authors propose that a posterior wall osteotomy is an acceptable option when confronted with this unusual situation.
\end{abstract}

Volume 8 Issue 5 - 2017

\author{
Sithombo Maqungo, Matthew Workman, \\ Michael Held, Mike Van Niekerk, Maritz \\ Laubscher \\ Orthopaedic Trauma Service, Groote Schuur Hospital, \\ University of Cape Town, South Africa
}

Correspondence: Sithombo Maqungo, P O Box 13665 , Mowbray 7705, South Africa, Tel +27 (0) 83234I 723, Fax +27 (0) 21 4472709 Email sithombo@msn.com

Received: July 17, 2017| Published: August 04, 2017

\section{Introduction}

In our practice we are frequently confronted with orthopaedic injuries from gunshots and gunshot injuries around the hip joint pose specific challenges.

Several complications are described following retained intra and juxta-articular bullets and bullet fragments. These include mechanical blockade to movement, direct cartilage destruction, synovitis, joint sepsis, lead arthropathy and even systemic lead poisoning (plumbism). ${ }^{1-4}$ It is therefore recommended that all intra and juxtaarticular bullets or bullet fragments should be removed. Removal can be performed via open arthrotomy or arthroscopically. ${ }^{5,6}$ Occasionally the location requires the usage of ingenious techniques.

We present a case of a patient with a bullet buried in the posterior acetabular wall. A decision was made to remove the bullet via a posterior wall acetabular osteotomy.

\section{Case report}

A 32 old male presented with a single gunshot wound to the right suprapubic area, with no exit. Radiographs revealed a bullet that appeared to be lodged in the left hip joint (Figures 1) A CT scan (Figures 2) and cystogram showed no associated injuries, with the bullet tract entering the quadrilateral surface of the left hip and the bullet buried in the posterior acetabular wall.

Due to it's juxta-articular location, the bullet needed to be removed. We did not consider approaches through the joint, arthroscopic or open, as the bullet was buried sub-chondrally. A clinical decision was made that the best approach would be to perform an osteotomy of the posterior wall as this would minimize damage to the acetabular articular cartilage and the capsule would be left undisturbed. We could however find no reference in the literature to this. We proceeded with the osteotomy through a Kocher-Langenbeck approach to the acetabulum. The bullet was removed and the osteotomy reduced and fixed with two interfragmentary screws and a buttress plate (Figure 3). A standard post-operative regime for posterior wall fixation was followed.
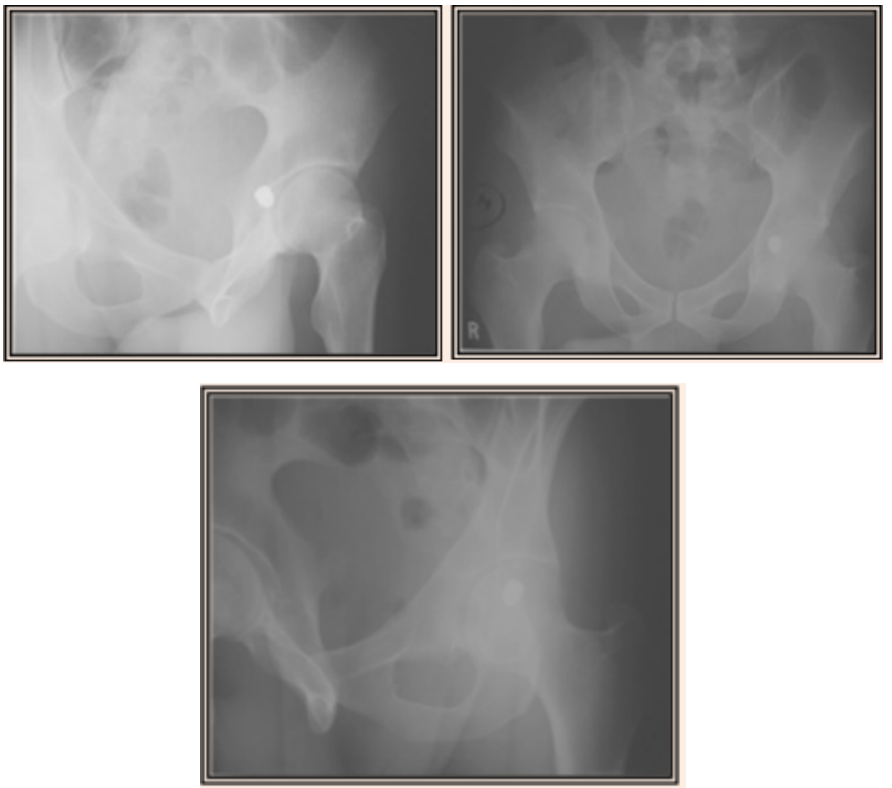

Figure I Radiographs at first presentation showing a bullet that appeared to be lodged in the left hip joint. 


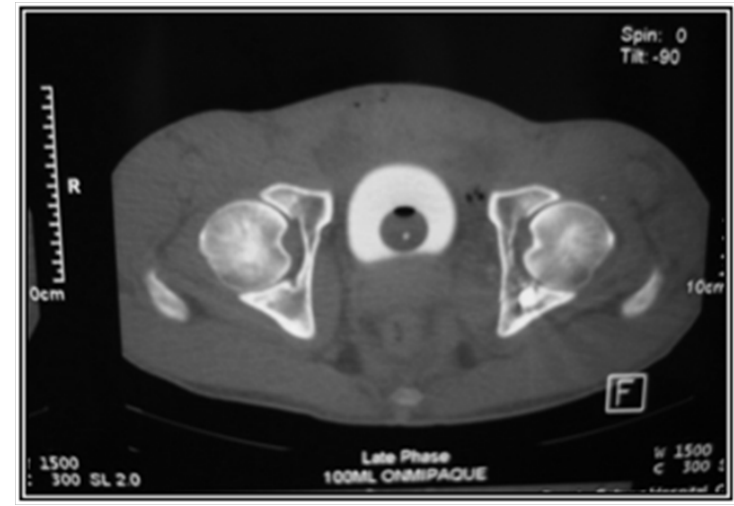

Figure 2 CT scan showed the bullet buried in the posterior acetabular wall.

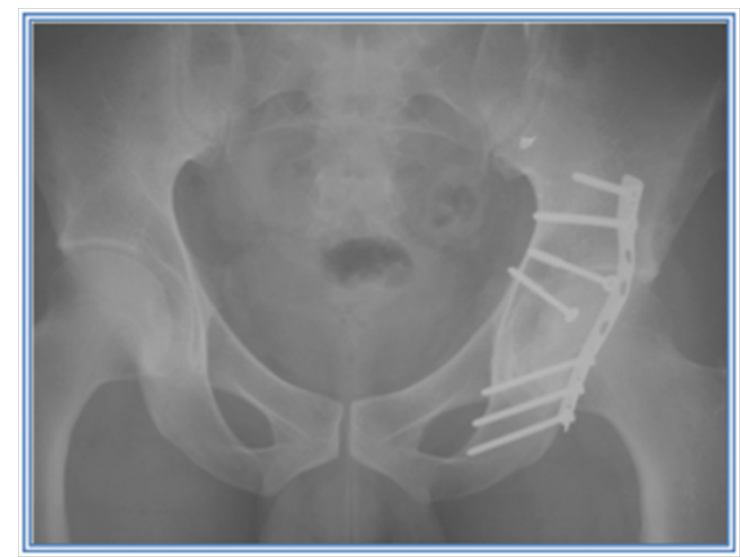

Figure 3 The osteotomy was reduced and fixed with two interfragmentary screws and a buttress plate.

At 2 years follow up the osteotomy site had united with no complications and the patient had regained a pain-free full range of motion, could walk unaided and had no limp.

\section{Conclusion}

We propose that a posterior wall osteotomy is an acceptable option when confronted with this unique situation.

\section{Acknowledgements}

None.

\section{Conflicts of Interest}

Each author certifies that he has no commercial associations that might pose a conflict of interest with the submitted article.

\section{References}

1. DeMartini J, Wilson A, Powell JS et al. Lead arthropathy and systemic lead poisoning from an intraarticular bullet. Am J Radiology. 2001;176(5):1144

2. Linden MA, Manton WI, Stewart RM et al. Lead poisoning from retained bullets. Ann Surg. 1982;195(3):305-313.

3. Bartlett CS1, Helfet DL, Hausman MR et al. Ballistics and gunshot wounds: effects on musculoskeletal tissues. J Am Acad Orthop Surg. 2000;8(1):21-36.

4. Thorsesby FP, Darlow HM The mechanism of primary infection of bullet wounds. Br J Surg. 1967;54(5):359-361.

5. Lee GH1, Virkus WW, Kapotas JS Arthroscopically Assisted Minimally Invasive Intraarticular Bullet Extraction: Technique, Indications, and Results. J Trauma. 2008;64(2):512-516.

6. Parisien JS, Esformes I The role of arthroscopy in the management of low-velocity gunshot wounds of the knee joint. Clin Orthop. 1984;185:207-213. 